\title{
Estudio arqueométrico del proceso pirometalúrgico usado entre 1903 y 1913 para la extracción de cobre en la fundición Santa Florentina (Chilecito, La Rioja, Argentina)
}

Nicolás M. Rendtorff", Martín E. Morosi" ${ }^{* *}$ y Eduardo L. Tavani $^{* * *}$
Recibido:

21 de febrero de 2019

Aceptado:

10 de junio de 2019

\section{Resumen}

El objetivo del presente trabajo fue dilucidar aspectos básicos y aplicados del proceso pirometalúrgico que fuera usado hace más de 100 años en la fundición Santa Florentina (Chilecito, provincia de La Rioja, Argentina) para extraer cobre a partir de minerales del yacimiento La Mejicana (Famatina). Con este propósito, formulamos las posibles reacciones que describen la obtención del metal, realizamos una exhaustiva caracterización fisicoquímica de la escoria, estimamos el desprendimiento gaseoso durante la oxidación de diversas sustancias, evaluamos el impacto de la minería en la deforestación regional y revisamos las causales que respaldaron la opinión de discontinuar el proyecto. La metodología experimental empleada comprendió: análisis químico, difracción de rayos $\mathrm{X}$, microscopía electrónica de barrido, espectroscopía de energía dispersiva de rayos $\mathrm{X}$, termogravimetría-análisis térmico diferencial y determinación de densidad. Los resultados aquí exhibidos nos permitieron establecer que la recuperación de cobre fue alta, recopilar detalles operativos de la tecnología implementada en aquella época, aportar evidencias sobre la contaminación ambiental producida por las actividades llevadas a cabo e identificar dos potenciales motivos que habrían impulsado a tomar la resolución de abandonar definitivamente el emprendimiento.

\footnotetext{
* Centro de Tecnología de Recursos Minerales y Cerámica (CETMIC) / Centro Científico Tecnológico (CCT) La Plata, Consejo Nacional de Investigaciones Científicas y Técnicas (CONICET) / Departamento de Química, Facultad de Ciencias Exactas, Universidad Nacional de La Plata (UNLP). Camino Centenario y 506, CC. 49, (CP 1897), Gonnet, Argentina. E-mail: rendtorff@cetmic.unlp.edu.ar

** Centro de Tecnología de Recursos Minerales y Cerámica (CETMIC) / Comisión de Investigaciones Científicas de la Provincia de Buenos Aires (CIC) / Facultad de Ciencias Naturales y Museo, Universidad Nacional de La Plata (UNLP). Camino Centenario y 506, CC. 49, (CP 1897), Gonnet, Argentina. E-mail: mmorosi@cetmic.unlp.edu.ar *** Centro de Tecnología de Recursos Minerales y Cerámica (CETMIC) / Comisión de Investigaciones Científicas de la Provincia de Buenos Aires (CIC). Camino Centenario y 506, CC. 49, (CP 1897), Gonnet, Argentina. E-mail: etavani@cetmic.unlp.edu.ar
}

\section{Palabras clave}

La Mejicana

Famatina

Enargita

Pirita

Escoria 


\title{
Archaeometric study of the pyrometallurgical process used between 1903 and 1913 for copper extraction in the Santa Florentina smelter (Chilecito, La Rioja, Argentina)
}

\begin{abstract}
Keywords

La Mejicana Famatina Enargite Pyrite Slag

The aim of the present work was to elucidate basic and applied aspects of the pyrometallurgical process which used more than 100 years ago in the Santa Florentina smelter (Chilecito, La Rioja province, Argentina) to extract copper from the minerals of $\mathrm{La}$ Mejicana deposit (Famatina). For this purpose, we formulated the possible reactions that describe the obtaining of the metal, we made a thorough physicochemical characterization of the slag, estimated the gas released during the oxidation of various substances, assessed the impact of mining on regional deforestation, and finally reviewed the causes that supported the opinion of discontinue the project. The experimental methodology used involved chemical analysis, X-ray diffraction, scanning electron microscopy, energydispersive X-ray spectroscopy, thermogravimetry-differential thermal analysis, and density determination. Results obtained allowed us to establish that the copper recovery rate was high; to gather operative details of the technology implemented at that time; to provide evidence on environmental pollution produced by the activities carried out, and to identify two potential reasons that would have prompted the decision to definitely abandon the venture.
\end{abstract}

\section{Introducción}

La Mejicana es un yacimiento polimetálico ubicado en el faldeo oriental del sistema de Famatina, provincia de La Rioja, Argentina. Su mineralización no es uniforme y varía enormemente de un nivel a otro. Las vetas (filones) de mayor importancia económica para los tres componentes más valiosos, según lo establecido por análisis químicos de muestreos hechos en las primeras décadas del siglo pasado, son Upulungos (U) y San Pedro (SP). Los contenidos promedio de cada una (U/SP) fueron: 30,8/72,6 kg ton ${ }^{-1}$ de $\mathrm{Cu}, 13 / 10 \mathrm{~g}^{-1}{ }^{-1}$ de Au y 125/59 $\mathrm{g}^{-1}{ }^{-1}$ de Ag (Angelelli, 1984; Lannefors, 1926).

A comienzos de 1903 se constituyó The Famatina Development Corporation "para adquirir una mina de cobre situada a $14.000 \mathrm{ft}(4267 \mathrm{~m})$ sobre el nivel del mar en las montañas de los Andes" (Anónimo, 1910, p. 375). Una vez comenzada la explotación, la empresa comprobó que la riqueza y naturaleza de los minerales procesados no satisfacían sus expectativas. Con la premisa de minimizar los perjuicios provocados por tales cuestiones se propusieron modificaciones al proyecto original. Inicialmente se adoptó una ruta en la cual parte del calor se generaba por combustión del azufre (régimen autógeno) y luego se abandonó (Anónimo, 1910; Leguizamon Pondal, 1919). Estudios geológicos recientes confirman que la especie mayoritaria aprovechable era enargita $\left(\mathrm{Cu}_{3} \mathrm{AsS}_{4}\right)$ y en mucha menor proporción calcopirita $\left(\mathrm{CuFeS}_{2}\right)$. A su vez, la pirita $\left(\mathrm{FeS}_{2}\right.$ ) fue un material de relleno bastante abundante (Angelelli, 1984; Bodenbender, 1916; Brodtkorb y Schalamuk, 1999; Lannefors, 1926; Leguizamon Pondal, 1919).

El horno de cuba con camisa de agua tuvo un uso muy difundido en el período que nos ocupa (primeras décadas del siglo XX) para concretar el mencionado proceso extractivo. Varias secciones de sus paredes estaban construidas en forma de cajas metálicas (acero) y por su interior circulaba el líquido refrigerante. Este diseño habitualmente conocido con el nombre de water jacket respondía, entre otros motivos, al hecho de que los ladrillos refractarios eran inestables. Durante la fusión solidificaba sobre las placas 
frías una fracción de la carga que actuaba como capa protectora contra la destrucción estructural (Kuzmín y Samojotski, 1986; Peters, 1907; Szczygiel Jordens y Torres Reyes, 1984).

Otro horno muy demandado por el sector industrial fue el de reverbero. A diferencia del anterior, la energía térmica se transmitía por reflexión en una bóveda de ladrillos refractarios. El hogar en que se quemaba el combustible -generalmente coque o carbón- estaba localizado a un costado del crisol que contenía el mineral y el fundente, en otras palabras el calentamiento procedía de manera indirecta (Evans y Saunders, 2017; Gill, 1989; Kuzmín y Samojotski, 1986; Leguizamon Pondal, 1914, 1919; Peters, 1907; Rosenqvist, 1987; Szczygiel Jordens y Torres Reyes, 1984; Wässman, 1926).

De los trabajos revisados, sólo uno (Leguizamon Pondal, 1919) describe de modo preciso el tipo de horno que fuera empleado para ejecutar cada paso del ciclo productivo en el establecimiento Santa Florentina (Chilecito): de reverbero-oxidación (tostación), de cuba-fusión y convertidor-obtención de cobre metálico (blíster). La operatoria acorde con estándares tradicionales posibilitaba oxidar los constituyentes reactivos de la mena y fundir los productos formados. Así se lograban dos fases, una rica en silicatos denominada escoria (densidad $=\sim 3,5 \mathrm{~g} \mathrm{~cm}^{-3}$ ) y otra compuesta básicamente por una mezcla de sulfuros llamada mata o eje (densidad $=\sim 5,0 \mathrm{~g} \mathrm{~cm}^{-3}$ ) (Kuzmín y Samojotski, 1986).

La distancia que media desde Upulungos (4.603 m s.n.m.) hasta Chilecito (1.125 m s.n.m.) es de $34,33 \mathrm{~km}$, el declive $3.478 \mathrm{~m}$ y la traza del camino/senda original resultaba sumamente escarpada (Bialet Massé, 1904; Leguizamon Pondal, 1914). En 1902 el gobierno nacional convocó a licitación para la construcción de un cable carril que conectara ambos puntos con el propósito de proveer un medio de transporte más eficaz en comparación al tradicional por animales de carga. El trayecto se componía de seis secciones con nueve estaciones y, los motores a vapor convenientemente localizados proporcionaban la tracción. Esta obra, una de las más importantes del mundo para la época, fue inaugurada el primero de enero de 1905. El diseño, construcción y montaje del referido sistema de traslación estuvo a cargo de la empresa A. Bleichert \& Co (Alemania). La capacidad de acarreo variaba con el sentido de circulación, ascendente 15 ton $\mathrm{h}^{-1} \mathrm{y}$ descendente 25 ton $\mathrm{h}^{-1}$. Para emplazar la segunda estación, distante $8,9 \mathrm{~km}$ de la cabecera en Chilecito y de su empalme con el ferrocarril, fue elegido un sitio próximo al horno de fundición que funcionaba desde la década anterior (Santa Florentina). El acceso a la instalación se hacía por un ramal secundario de muy corto recorrido (Angelelli, 1984; Bazán, 2017; Bodenbender, 1916; Hermitte, 1917; Lannefors, 1926). En la Figura 1 se aprecia la topografía predominantemente montañosa de la región y las distancias relativas entre los tres lugares de interés.

La arqueometría es una disciplina que permite responder interrogantes acerca de tecnologías desarrolladas a lo largo del tiempo y sus correspondientes relaciones contextuales (Becerra, Nieva y Angiorama, 2014; Ortega, Zuluaga, Alonso y Olaetxea, 2005; Quiroga, Chiavazza, Lascalea y Gurrito, 2017; Setti, Lanfranchi, Cultrone y Marinoni, 2012; Thibodeau et al., 2007). De acuerdo con los antecedentes mencionados, en esta contribución se propuso dilucidar aspectos metalúrgicos que conduzcan a una descripción detallada de las transformaciones que ocurrieron en las sucesivas etapas del emprendimiento, efectuar la caracterización fisicoquímica de la escoria generada por el establecimiento Santa Florentina, cuantificar mediante cálculos estequiométricos la polución atmosférica ocasionada en un año de trabajo, evaluar el impacto de la minería en el desmonte de bosques nativos del oeste riojano e identificar posibles argumentos capaces de explicar la decisión de abandonar la explotación. Obviamente, dicha investigación retrospectiva es parcial ya que no se dispuso de la mata producida, ni las materias primas utilizadas. Para el pertinente tratamiento sólo se contó con la citada 


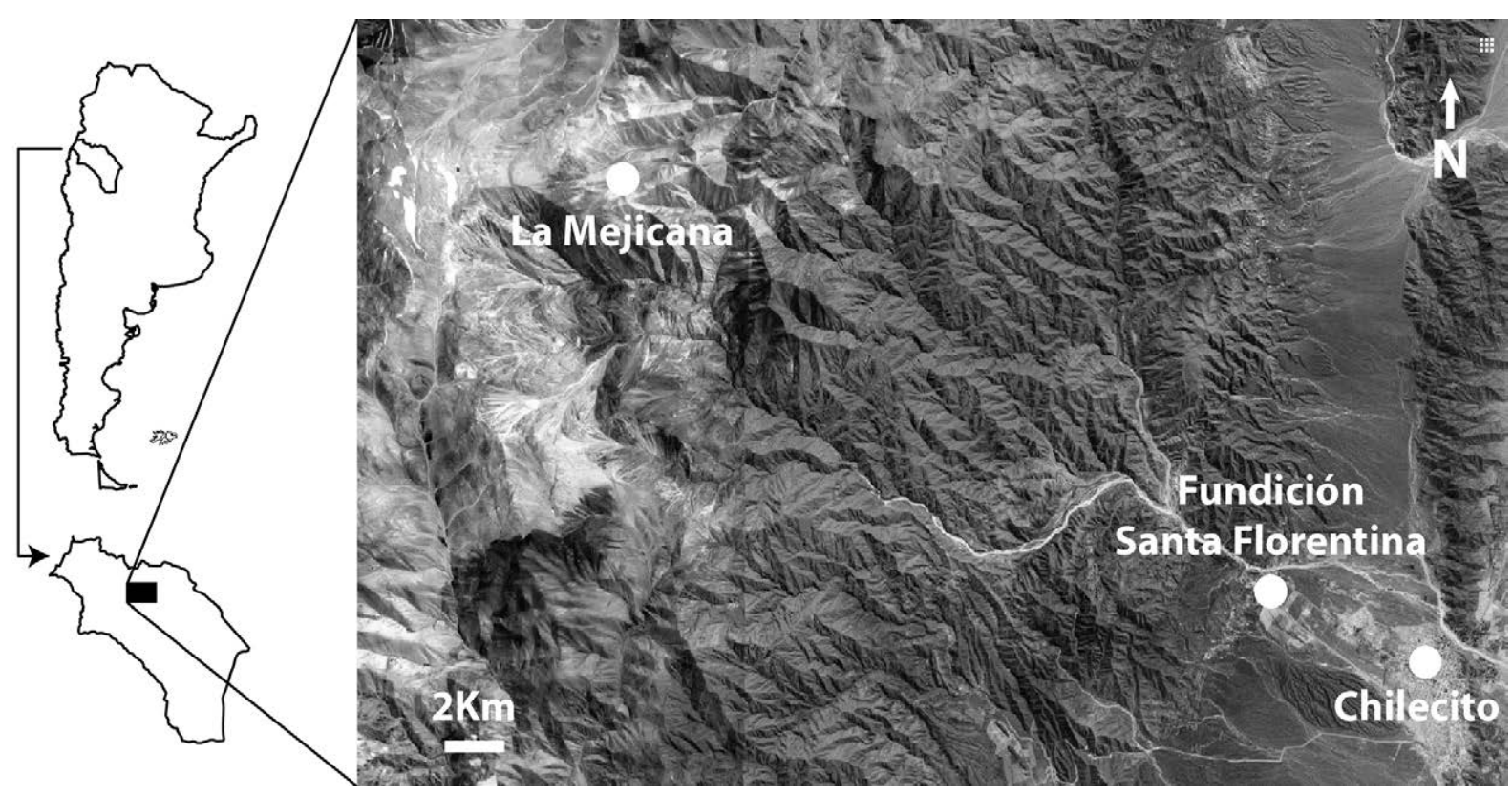

Figura 1. Ubicación de los sitios relevantes (Google Earth Pro, DigitalGlobe, CNES/Airbus) del complejo minero metalúrgico.

escoria y unos pocos, en oportunidades contradictorios, reportes contemporáneos al lapso que abarca la revisión.

\section{Metodología}

La muestra fue extraída del sector medio de la pila central, cuya disposición se observa en la Figura 2. Los ensayos llevados a cabo fueron: análisis químico, difracción de rayos $\mathrm{X}$ (DRX), microscopía electrónica de barrido (MEB) con espectroscopía de energía dispersiva de rayos X (EDS), termogravimetría-análisis térmico diferencial (TG-ATD) y evaluación de la densidad. En la Tabla 1 se indica la composición química de la escoria en $\%$ peso/peso $(\% \mathrm{p} / \mathrm{p})$.

Las determinaciones analíticas se realizaron empleando técnicas estándares de volumetría, gravimetría y absorción/emisión atómica con un instrumento Buck Scientific, 210 VGP. El estudio por DRX fue ejecutado con un equipo Philips $3 \mathrm{~kW}$ $\mathrm{X}$ 'Pert, usando radiación $\mathrm{K} \alpha$ del $\mathrm{Cu}$ y filtro de Ni. Para las valoraciones por medio de MEB-EDS se dispuso de un microscopio FEI-Quanta 200 acoplado a la sonda de microanálisis EDS-EDAX Apollo X. El metalizado se practicó con oro. Los TG y ATD fueron hechos simultáneamente en el módulo Rigaku Thermo plus EVO2, TG 8121, estimándose la pérdida de peso $(\% \mathrm{pdp})$ entre 30 y $1000{ }^{\circ} \mathrm{C}$. Las condiciones operativas fueron: velocidad de calentamiento $10^{\circ} \mathrm{C} \mathrm{min}^{-1}$ bajo atmósfera dinámica de nitrógeno (inerte) y flujo gaseoso $6,0 \mathrm{~L} \mathrm{~min}^{-1}$. Finalmente, la densidad fue medida por pesada.

\section{Reacciones formuladas para describir la obtención del cobre}

Las menas ricas en azufre, $\sim 35 \% \mathrm{p} / \mathrm{p}$ del elemento $\left(\mathrm{FeS}_{2}, \mathrm{Cu}_{3} \mathrm{AsS}_{4}, \mathrm{CuFeS}_{2}\right)$, se funden sin agregar coque o con una adición mínima $(<2 \%)$ y el proceso se denomina pirítico (régimen autógeno). Cuando se aumenta el porcentaje de coque hasta $\sim 6 \% \mathrm{p} / \mathrm{p}$, la fusión se llama semipirítica (Kuzmín y Samojotski, 1986). En atmósferas libre o con escasa presencia de oxígeno se altera el balance anterior ya que a partir de $600-700{ }^{\circ} \mathrm{C}$, 


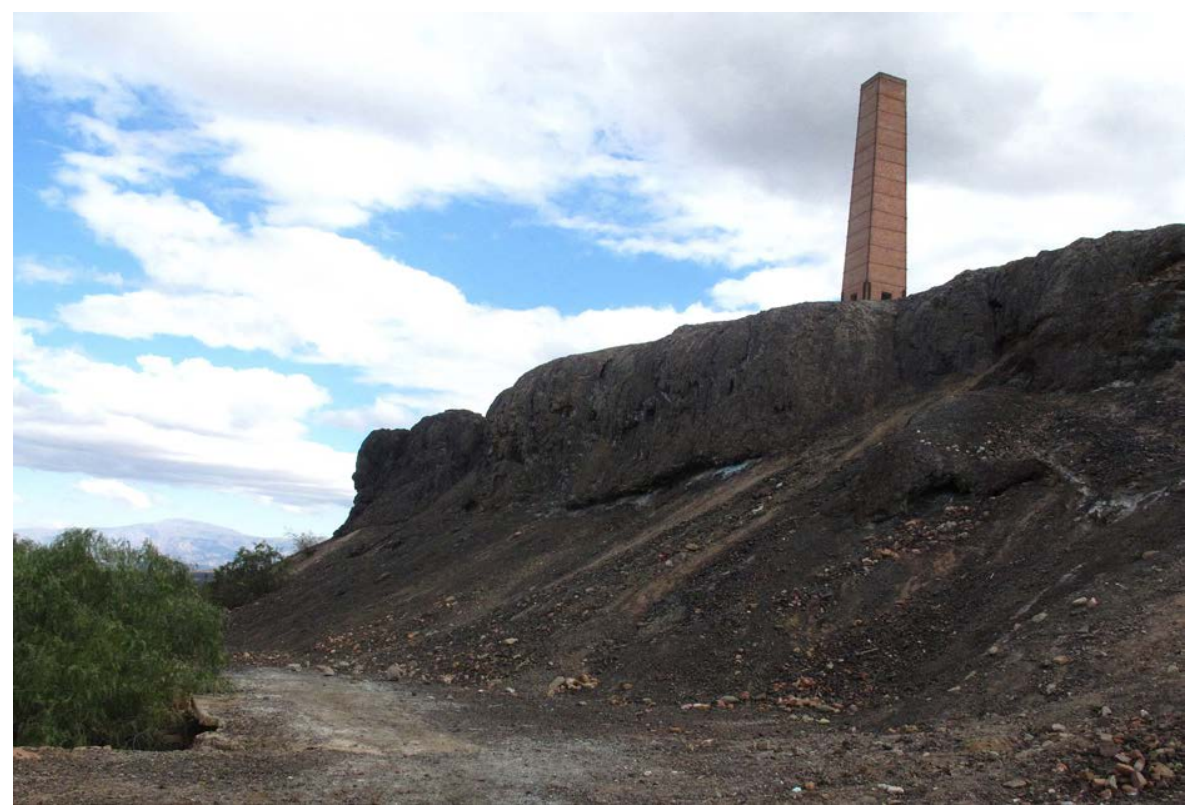

Figura 2. Vista panorámica del depósito de escoria generado por la actividad llevada a cabo en Santa Florentina, Chilecito, La Rioja.

\begin{tabular}{|l|c|}
\cline { 2 - 2 } \multicolumn{1}{c|}{} & \% p/p \\
\hline $\mathrm{SiO}_{2}$ & 41,85 \\
$\mathrm{Al}_{2} \mathrm{O}_{3}$ & 9,99 \\
$\mathrm{Fe}_{2} \mathrm{O}_{3}$ & 4,48 \\
$\mathrm{FeO}$ & 12,92 \\
$\mathrm{CaO}$ & 14,27 \\
$\mathrm{MgO}$ & 5,98 \\
$\mathrm{Na}_{2} \mathrm{O}$ & 0,19 \\
$\mathrm{~K}_{2} \mathrm{O}$ & 0,56 \\
$\mathrm{CuO}^{2}$ & 0,26 \\
$\mathrm{~S}_{2} / \mathrm{S}^{2-}$ & $\mathrm{nd}$ \\
$\mathrm{SO}_{2-}$ & 5,22 \\
$\mathrm{pdp}_{4} \mathrm{C}\left(900^{\circ} \mathrm{C}\right)$ & 4,23 \\
\hline
\end{tabular}

Tabla 1. Análisis químico de la muestra. El porcentaje de los elementos, a excepción del azufre, fue expresado como óxido. Referencias: nd: no detectado; $p d p-C$ : pérdida de peso por calcinación a $900^{\circ} \mathrm{C}$.

casi mitad de la temperatura de trabajo $\sim 1200{ }^{\circ} \mathrm{C}$, el bisulfuro de hierro comienza a descomponerse (ecuación (1)) (Jovanovic, 1989; Menéndez, Tavani y Nolasco, 2003; Peters, 1907).

El azufre condensa parcialmente (puntos de ebullición/fusión $\sim 445^{\circ} \mathrm{C} / \sim 119{ }^{\circ} \mathrm{C}$ ) (Hammond, 1981) en las partes frías del horno y si más tarde se oxida, su aporte de energía no se aprovecha para calefaccionar la carga. Según esta pirólisis, se reduce el poder calorífico de la pirita y a los efectos prácticos sólo se debe tomar en cuenta la 
pirrotina (FeS) (ecuaciones (2) y (3)). Las variaciones de energía libre a $700{ }^{\circ} \mathrm{C}$ confirman el fuerte carácter exotérmico de las transformaciones del sulfuro de hierro: $\Delta \mathrm{G}_{700^{\circ} \mathrm{C}} \sim-390$ $\mathrm{kJ} \mathrm{mol}^{-1}$ para la ecuación (2) y $\Delta \mathrm{G}_{700^{\circ} \mathrm{C}} \sim-475 \mathrm{~kJ} \mathrm{~mol}^{-1}$ para la ecuación (3) (Rosenqvist, 1987). En caso de ocurrir la calcinación en presencia de abundante oxígeno, se producen otras reacciones que mejoran la performance térmica (ecuaciones (4)-(5)).

La ejecución del ciclo productivo con minerales extraídos de ambas vetas no tuvo el comportamiento esperado con relación a su contenido de azufre, razón por la que se propuso hacer una oxidación previa (Anónimo, 1910). Las menas pobres en cobre (Upulungos) eran tostadas directamente en la mina a cielo abierto, mientras que las de mayor riqueza (San Pedro) se trasladaban a Santa Florentina para cumplir con dicho tratamiento en un horno de reverbero (Leguizamon Pondal, 1914, 1919). La innovación se hizo efectiva durante los primeros meses de 1911 y los resultados logrados inducen a pensar que dicha modificación incrementó el rendimiento del procedimiento.

El oxígeno tiene mayor afinidad con el hierro y el arsénico que con el cobre (Barone, Gazzoli, Lick, Schalamuk y Botto, 2011; Gill, 1989; Szczygiel Jordens y Torres Reyes, 1984). Por tal motivo, se asume que la oxidación del FeS sucedía de modo casi cuantitativo (ecuaciones (2)-(3)) y la de $\mathrm{Cu}_{3} \mathrm{AsS}_{4}$ en menor proporción. Si la presión de oxígeno era suficiente se formaba sulfuro cuproso $\mathrm{Cu}_{2} \mathrm{~S}$ (ecuación (6)) (Mihajlovic, Strbac, Zivkovic, Kovacevic y Stehernik, 2007) o de acuerdo con una propuesta más reciente digenita $\mathrm{Cu}_{9} \mathrm{~S}_{5}$ (ecuación (7)) (Barone et al., 2011). Cabe aclarar que la velocidad de insuflado del aire (oxígeno) es crítica, porque en caso de ser muy alta enfriaría parcialmente la carga. Respecto al arsénico y con base en datos termodinámicos, se puede señalar que el elemento volatilizado adoptaba la fórmula de $\mathrm{As}_{4} \mathrm{O}_{6}$ (dímero del monómero $\mathrm{As}_{2} \mathrm{O}_{3}$ ) (Aracena Caipa, 2013; Mihajlovic et al., 2007).

La fusión de la mena oxidada se llevaba a cabo en un horno de cuba. Una vez alcanzado el estado líquido, la plata (punto de fusión $=962{ }^{\circ} \mathrm{C}$, densidad $=10,5 \mathrm{~g} \mathrm{~cm}^{-3}$ ) y el oro (punto de fusión $=1064{ }^{\circ} \mathrm{C}$, densidad $\left.=19,3 \mathrm{~g} \mathrm{~cm}^{-3}\right)$ (Hammond, 1981) se concentraban en la mata. Hacia dicha fase también difundía parte del FeS (punto de fusión = 1193$1199^{\circ} \mathrm{C}$, densidad $=4,74 \mathrm{~g} \mathrm{~cm}^{-3}$ ) (Hammond, 1981) que aún permanecía disuelto, es decir aquel que no había participado como reactivo de las ecuaciones (2)-(3). Con este proceder resultaba una mezcla de sulfuros $\left(\mathrm{Cu}_{2} \mathrm{~S}+\mathrm{FeS}\right)$ con trazas de metales preciosos que se separaba de la escoria por gravedad (Anónimo, 1914; Leguizamon Pondal, 1914, 1919). La notación precedente incluye exclusivamente $\mathrm{al} \mathrm{Cu}_{2} \mathrm{~S}$ en carácter de sustancia portadora del cobre conforme a las precisiones vertidas en el trabajo de Leguizamon Pondal (1919). Una ventaja adicional de la tecnología descrita fue que se redujo casi hasta su extinción el campo de aplicación de la amalgamación, con el lógico beneficio ambiental que ello implicaba (Cooke, Wolfe y Hobbs, 2009).

$$
\begin{aligned}
& 2 \mathrm{FeS}_{2}(\mathrm{~s}) \rightarrow 2 \mathrm{FeS}(\mathrm{s})+\mathrm{S}_{2}(\mathrm{~g}) \\
& 2 \mathrm{FeS}(\mathrm{s})+3 \mathrm{O}_{2}(\mathrm{~g}) \rightarrow 2 \mathrm{SO}_{2}(\mathrm{~g})+2 \mathrm{FeO}(\mathrm{s}) \\
& 4 \mathrm{FeS}(\mathrm{s})+7 \mathrm{O}_{2}(\mathrm{~g}) \rightarrow 4 \mathrm{SO}_{2}(\mathrm{~g})+2 \mathrm{Fe}_{2} \mathrm{O}_{3}(\mathrm{~s}) \\
& 2 \mathrm{FeS}_{2}(\mathrm{~s})+5 \mathrm{O}_{2}(\mathrm{~g}) \rightarrow 4 \mathrm{SO}_{2}(\mathrm{~g})+2 \mathrm{FeO}(\mathrm{s}) \\
& 4 \mathrm{FeS}_{2}(\mathrm{~s})+11 \mathrm{O}_{2}(\mathrm{~g}) \rightarrow 8 \mathrm{SO}_{2}(\mathrm{~g})+2 \mathrm{Fe}_{2} \mathrm{O}_{3}(\mathrm{~s}) \\
& 4 \mathrm{Cu}_{3} \mathrm{AsS}_{4}(\mathrm{~s})+13 \mathrm{O}_{2}(\mathrm{~g}) \rightarrow 6 \mathrm{Cu}_{2} \mathrm{~S}(\mathrm{~s})+\mathrm{As}_{4} \mathrm{O}_{6}(\mathrm{~g})+10 \mathrm{SO}_{2}(\mathrm{~g}) \\
& 12 \mathrm{Cu}_{3} \mathrm{AsS}_{4}(\mathrm{~s})+37 \mathrm{O}_{2}(\mathrm{~g}) \rightarrow 4 \mathrm{Cu}_{9} \mathrm{~S}_{5}(\mathrm{~s})+3 \mathrm{As}_{4} \mathrm{O}_{6}(\mathrm{~g})+28 \mathrm{SO}_{2}(\mathrm{~g})
\end{aligned}
$$

El refinado de $\mathrm{Cu}$ tiene una ley que depende de la técnica metalúrgica usada, variando dentro de un amplio rango entre 16 y 60\% p/p (Kuzmín y Samojotski, 1986). En la Tabla 2 se indican tres concentraciones de sendas matas, pero ninguna de las referencias especifica claramente el momento en que fueron elaboradas. Aun cuando los valores mencionados exhiben una enorme disparidad, las dos primeras publicaciones coinciden 


\begin{tabular}{|c|c|c|}
\hline $\mathrm{Cu}(\% \mathrm{p} / \mathrm{p})$ & $\mathrm{Ag} / \mathrm{Au}\left(\mathrm{g} \mathrm{ton}^{-1}\right)$ & Referencia \\
\hline 61,24 & $7340 / 135$ & Anónimo, 1914 \\
28 & - & Leguizamon Pondal, 1914 \\
$18-20$ & - & Hermitte, 1917 \\
\hline
\end{tabular}

Tabla 2. Ley de Cu de tres matas obtenidas en Santa Florentina.

en afirmar que mediante la utilización del convertidor fue posible obtener un producto con alta pureza (blíster) de Cu: 96\% (Anónimo, 1914) o 98\% p/p (Leguizamon Pondal, 1914). Este cambio de estrategia, implementado a inicios de 1911, le confería sin lugar a dudas mayor utilidad al emprendimiento. La falta de precisión de la información disponible induce a cometer errores de apreciación que ciertamente le restan autenticidad al relato.

La reducción del catión cuproso $\left(\mathrm{Cu}_{2} \mathrm{~S}\right)$ a cobre metálico $(96-98 \% \mathrm{de} \mathrm{Cu})$ fue otra de las modificaciones aplicadas en el diagrama de flujo de Santa Florentina, acción que se ejecutaba por soplado de aire (Díaz Marinovic, 2012; Leguizamon Pondal, 1919; Vera Giglio, 2007). Primero se eliminaba el FeS (ecuación (2)), mientras que en esta etapa la conversión del $\mathrm{Cu}_{2} \mathrm{~S}$ era mínima. A su vez, el $\mathrm{FeO}$ reaccionaba con el $\mathrm{SiO}_{2}$ que recubría internamente el horno (ecuación (8)). Esta cobertura se lograba con sílice molida y tenía varias funciones: anticorrosivo de la carcasa metálica, aislante térmico y agente escorificante del óxido ferroso. Cada capa posibilitaba efectuar hasta doce coladas, posteriormente se requería su reposición (Leguizamon Pondal, 1919). Si se continuaba con el agregado de aire (oxígeno) procedía la oxidación del sulfuro cuproso (ecuación (9)). Una vez agotado el FeS, se combinaban el $\mathrm{Cu}_{2} \mathrm{~S}$ remanente y $\mathrm{Cu}_{2} \mathrm{O}$ (ecuación (10)). Las transformaciones detalladas son altamente exotérmicas, razón por la cual se requería una fuente de calor externa muy reducida.

$$
\begin{aligned}
& 2 \mathrm{FeO}(\mathrm{s})+\mathrm{SiO}_{2}(\mathrm{~s}) \rightarrow \mathrm{Fe}_{2} \mathrm{SiO}_{4}(\mathrm{~s}) \\
& 2 \mathrm{Cu}_{2} \mathrm{~S}(\mathrm{~s})+3 \mathrm{O}_{2}(\mathrm{~g}) \rightarrow 2 \mathrm{Cu}_{2} \mathrm{O}(\mathrm{s})+2 \mathrm{SO}_{2}(\mathrm{~g}) \\
& 2 \mathrm{Cu}_{2} \mathrm{O}(\mathrm{s})+\mathrm{Cu}_{2} \mathrm{~S}(\mathrm{~s}) \rightarrow 6 \mathrm{Cu}(\mathrm{s})+\mathrm{SO}_{2}(\mathrm{~g}) \\
& 2 \mathrm{CaO}(\mathrm{s})+2 \mathrm{SO}_{2}(\mathrm{~g})+\mathrm{O}_{2}(\mathrm{~g}) \rightarrow 2 \mathrm{CaSO}_{4}(\mathrm{~s})
\end{aligned}
$$

\section{Caracterización fisicoquímica de la escoria}

El estudio arqueométrico de este trabajo comprendió básicamente la caracterización fisicoquímica de la escoria (Rendtorff, Morosi y Tavani, 2018). Su análisis químico (Tabla 1) nos revela que la pérdida de cobre durante la separación de fases fue mínima en comparación con el contenido de la mena, 2,56-2,79\% p/p de Cu para Upulungos y 7,30-7,85\% p/p de Cu para San Pedro (Anónimo, 1910; Hermitte, 1917). En tanto, la no detección de $S_{2}^{2-} / S^{2-}$ evidencia el agotamiento de enargita y de pirita, probando además que los sulfuros $\left(\mathrm{Cu}_{2} \mathrm{~S}+\mathrm{FeS}\right)$ después de formados permanecían en la mata. Resumiendo, la valoración cuantitativa realizada es un modo sencillo para establecer la eficacia de la secuencia operativa que fuera empleada. El hierro representa un caso particular ya que fue el único elemento metálico mayoritario determinado con dos estados de oxidación, divalente y trivalente. Un porcentaje de hierro(II) menor al 30-40\%, y el hierro(III), aún en mínima cantidad, son variables que por sí solas elevan el punto de fusión de la escoria (Peters, 1907).

En el difractograma de la muestra (Figura 3) fueron identificados: yeso $\left(\mathrm{CaSO}_{4} \cdot 2 \mathrm{H}_{2} \mathrm{O}\right)$, a-cuarzo $\left(\mathrm{SiO}_{2}\right)$, monticellita $(\mathrm{CaMgSiO})$ y augita $\left((\mathrm{Ca}, \mathrm{Mg}, \mathrm{Fe})_{2}(\mathrm{Si}, \mathrm{Al})_{2} \mathrm{O}_{6}\right)$. Se trata de minerales que han sido citados como constituyentes secundarios posibles (ganga) del 


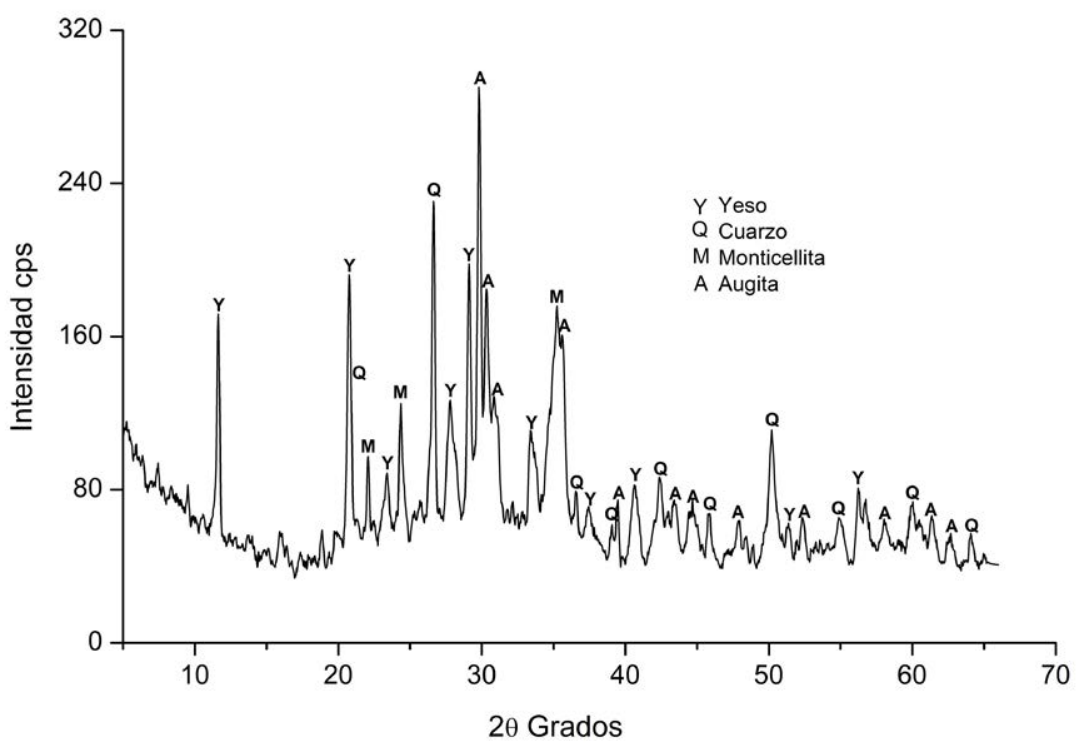

Figura 3. Difractograma de la muestra.

yacimiento (Angelelli, 1984; Bodenbender, 1916; Brodtkorb y Schalamuk, 1999). El primero se descompone por calentamiento a $165^{\circ} \mathrm{C}\left(\mathrm{CaSO}_{4} \cdot 2 \mathrm{H}_{2} \mathrm{O}(\mathrm{s}) \rightarrow \mathrm{CaSO}_{4}(\mathrm{~s})+2\right.$ $\mathrm{H}_{2} \mathrm{O}(\mathrm{g})$ ), en cambio los tres restantes no tienen grupos funcionales removibles por esta vía (Mackenzie, 1970). La sal de calcio anhidra se re-hidrata en ambientes húmedos y acorde al tiempo extremadamente largo de permanencia, se consideró razonable no haber encontrado el producto libre de $\mathrm{H}_{2} \mathrm{O}$. El promedio anual de precipitación oscila entre 100 y 200 mm (Varela, Parrado y Buedo, 2015).

Inicialmente, se supuso que mientras se efectuaba la fusión las cuatro sustancias nombradas se acumulaban en la escoria conforme a su afinidad. Sin embargo, algunos antecedentes temáticos aportaron una visión diferente. Así, la carga del horno de cuba se hacía con la alimentación previamente tostada, molida y mezclada con cal apagada para su aglomeración (Leguizamon Pondal, 1919). Con igual metodología, los finos colectados del tragante eran reciclados (Anónimo, 1914). La oxidación del dióxido de azufre y su combinación con el metal alcalinotérreo (ecuación (11)) (Menéndez et al., 2003; Peters, 1907) conlleva la síntesis de $\mathrm{CaSO}_{4}$ y nos ofrece una explicación alternativa acerca del origen de esta especie. Similar reparo también se advierte para el cuarzo, ya que prescindiendo de su presencia inicial fue agregado con carácter de fundente (Leguizamon Pondal, 1919; Wässman, 1926).

El análisis por difracción de rayos $\mathrm{X}$ no detectó wüstita (FeO) (Rovira y Burillo, 2005) (punto de fusión $=1369 \pm 1{ }^{\circ} \mathrm{C}$ ) (Hammond, 1981), hematita $\left(\mathrm{Fe}_{2} \mathrm{O}_{3}\right)$ (punto de fusión $=1565^{\circ} \mathrm{C}$ ) (Hammond, 1981) ni fayalita $\left(\mathrm{Fe}_{2} \mathrm{SiO}_{4}\right)$ (ecuaciones (2)-(5), (8)). La ausencia de picos de difracción característicos de estos componentes - falta de ordenamientos atómicos de largo alcance- ratificó la opinión de que si se encuentran en el sistema, solidificaron con arreglos cuyas celdas unitarias se repiten unas pocas veces (sólidos amorfos). El más relevante de los tres es silicato ferroso, isomorfo con monticellita, por ser un producto ( $\sim 30 \% \mathrm{de} \mathrm{SiO}_{2}$ (tridimita) y $\sim 70 \%$ de $\left.\mathrm{FeO}\right)$ que según lo señalado en el diagrama de fases de ambos óxidos tiene un punto de fusión próximo a $1200^{\circ} \mathrm{C}$ (Díaz Marinovic, 2012). Por otra parte, se descartó que hubiera magnetita (punto de fusión $=1594 \pm 5^{\circ} \mathrm{C}$ ) (Hammond, 1981) ya que ninguna partícula fue atraída por imanes de regular intensidad y la relación de concentración $\mathrm{FeO}: \mathrm{Fe}_{2} \mathrm{O}_{3}(12,92: 4,48=2,88)$ no es la del referido mineral $\mathrm{FeO} . \mathrm{Fe}_{2} \mathrm{O}_{3}(71,85: 159,7=0,45)$. La obtención de la fase cristalina 
$\mathrm{Fe}_{2} \mathrm{O}_{3}$ (DRX) se logró luego de calentar la muestra en estufa, bajo atmósfera estática de aire, a $1000{ }^{\circ} \mathrm{C}$ y enfriar a temperatura ambiente. El color de la alícuota así calcinada viró de gris oscuro a rojo tenue. $\mathrm{Al}$ adoptar estos compuestos estructuras indefinidas se produce una escoria más homogénea, o sea de mayor fluidez. Para concluir, el más alto porcentaje de hierro(II) comparado al de hierro(III) (Tabla 1) indica que la fusión fue efectuada en una atmósfera oxidante tenue.

La observación por MEB del material molido manualmente reveló que algunos granos con tamaños superiores a $200 \mu \mathrm{m}$, se distinguen por tener partículas sobrepuestas bastantes pequeñas $(4 \mu \mathrm{m}$ o menos). Tales ejemplares exhiben una textura apreciablemente irregular, notándose que sólo unos pocos están totalmente cubiertos. De acuerdo con dicha morfología, se juzgó conveniente revisar su composición química (EDS-EDAX). La valoración tuvo una resolución que llamó la atención y que significó haber encontrado carbono en numerosos especímenes. Su origen se atribuyó primariamente a la calcita $\left(\mathrm{CaCO}_{3}\right)$, que agregada junto con sílice, posibilitaba elaborar una escoria polibásica $\left(\mathrm{SiO}_{2}-\mathrm{CaO}-\mathrm{FeO}\right)$ (Peters, 1907), actualmente denominada olivina (Vera Giglio, 2007). El precedente que sustenta esta afirmación hace mención a la cantidad de caliza utilizada para producir ese fin (Wässman, 1926). A modo ilustrativo se indica una medición realizada al grano en la Figura 4 (\% p/p): 26,86 O; $8,82 \mathrm{C} ; 6,24 \mathrm{Si} ; 55,08 \mathrm{Ca} ; 1,68 \mathrm{Fe} ; 0,85 \mathrm{Al}$ y $0,48 \mathrm{~K}$. Al dividir el porcentaje de cada elemento por su respectivo peso atómico y descartar los tres valores minoritarios ( $\mathrm{Fe}$; $\mathrm{Al} ; \mathrm{K})$, ya que carecen de significación, se tiene: $1,68 \mathrm{O} ; 0,73 \mathrm{C} ; 0,22 \mathrm{Si}$; 1,38 Ca.

Un argumento contradictorio al hallazgo expuesto fue que el horno alcanzaba una temperatura muy superior $\left(1200-1300^{\circ} \mathrm{C}\right)$ (Peters, 1907) a la cual ocurre la pirólisis de calcita $\left(\sim 900^{\circ} \mathrm{C}\right)$ (Mackenzie, 1970$)$, consecuentemente la fuente del C sería incierta. El $\mathrm{CO}_{2}$ liberado $\left(\mathrm{CaCO}_{3}(\mathrm{~s}) \rightarrow \mathrm{CO}_{2} \uparrow(\mathrm{g})+\mathrm{CaO}(\mathrm{s})\right)$ en la interfase de reacción debía difundir a través de la capa de $\mathrm{CaO}$ naciente (Gaviría, Quattrini, Fouga, Bohé y Pasquevich, 2005). Dicha base tiene una enorme afinidad con $\mathrm{SiO}_{2}$, por lo que sería aceptable inferir que los dos óxidos se combinan para formar principalmente silicato de calcio $\left(\mathrm{Ca}_{2} \mathrm{SiO}_{4}\right)$ (Peters, 1907). Con un criterio cinético, se afirma que la sal se comporta como una barrera a la prosecución del desprendimiento de $\mathrm{CO}_{2}$. En caso de contar simultáneamente con $\mathrm{MgO}$ disuelto, el producto es un silicato doble de calcio y magnesio (monticellita), resultado ya transmitido. La otra interpretación, considerada aún más probable, estaría asociada con la re carbonatación de la escoria líquida durante su enfriamiento. Los estudios por DRX y TG-ATD no aportaron ninguna evidencia al respecto.

Varias de las aseveraciones aquí formuladas se sustentan en la información que surge del análisis térmico de la muestra (Figura 5). En el intervalo comprendido de 70 hasta $110^{\circ} \mathrm{C}$ se produjo la evaporación de humedad $(\sim 0,6 \% \mathrm{p} / \mathrm{p})$. La siguiente transformación concluyó a $150^{\circ} \mathrm{C}$, con una pdp más brusca que la anterior $\sim 2,4 \%$, y se relacionó con la liberación de agua del $\mathrm{CaSO}_{4} \cdot 2 \mathrm{H}_{2} \mathrm{O}$. Cabe acotar que los dos eventos reseñados fueron endotérmicos. A $789^{\circ} \mathrm{C}$ se determinó un pico exotérmico que correspondería a la desvitrificación de la escoria. La evolución más difícil de descifrar fue la pérdida de peso sostenida entre 150 y $1000^{\circ} \mathrm{C}(\sim 2,1 \%)$. Por presentar un porcentaje pequeño en una matriz tan compleja no se consiguieron pruebas al examinar sus elementos constituyentes y la mineralogía. Dicho fenómeno se debería a la volatilización de sustancias retenidas durante el enfriamiento (Mackenzie, 1970).

El índice de basicidad $\left(\mathrm{IB}=(\mathrm{MgO}+\mathrm{CaO}) /\left(\mathrm{Al}_{2} \mathrm{O}_{3}+\mathrm{SiO}_{2}\right)=0,39\right)$ y la densidad $(\delta$ $=3,30 \mathrm{~g} \mathrm{~cm}^{-3}$ ) completan la caracterización fisicoquímica de la escoria (Nazer, Payá, Borrachero y Monzó, 2016). Un hecho bien conocido se refiere al comportamiento dual (anfótero) del $\mathrm{Al}_{2} \mathrm{O}_{3}$ (Leguizamon Pondal, 1919; Peters, 1907), pero en la fórmula escrita previamente se concibe que actúa exclusivamente con carácter ácido. Sin embargo, 


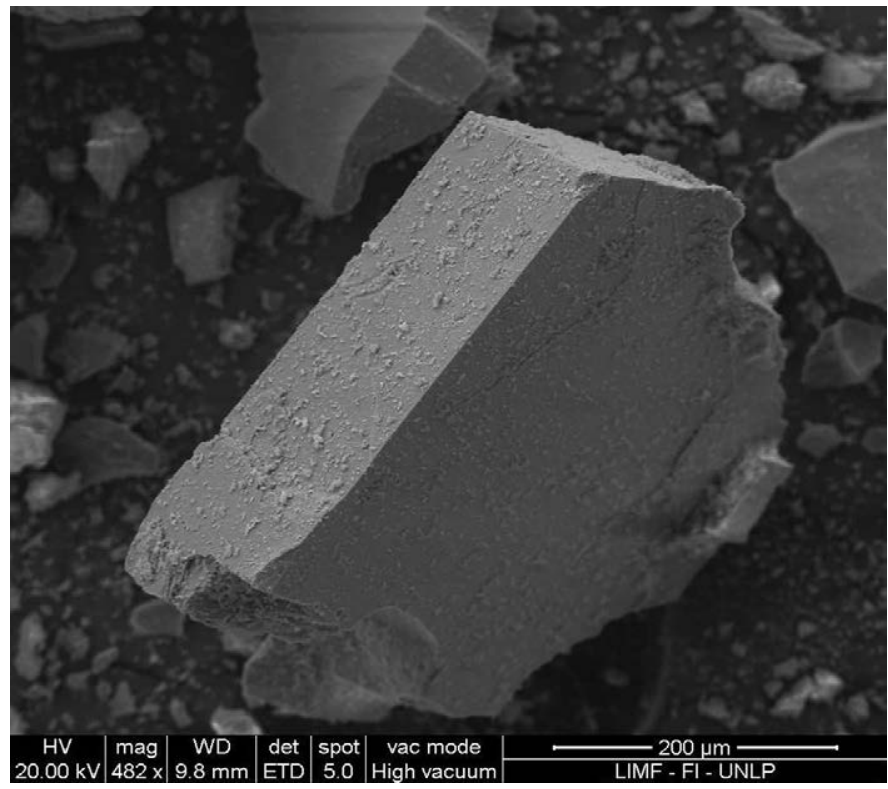

Figura 4. Micrografía del grano que fue analizado por EDS-EDAX.

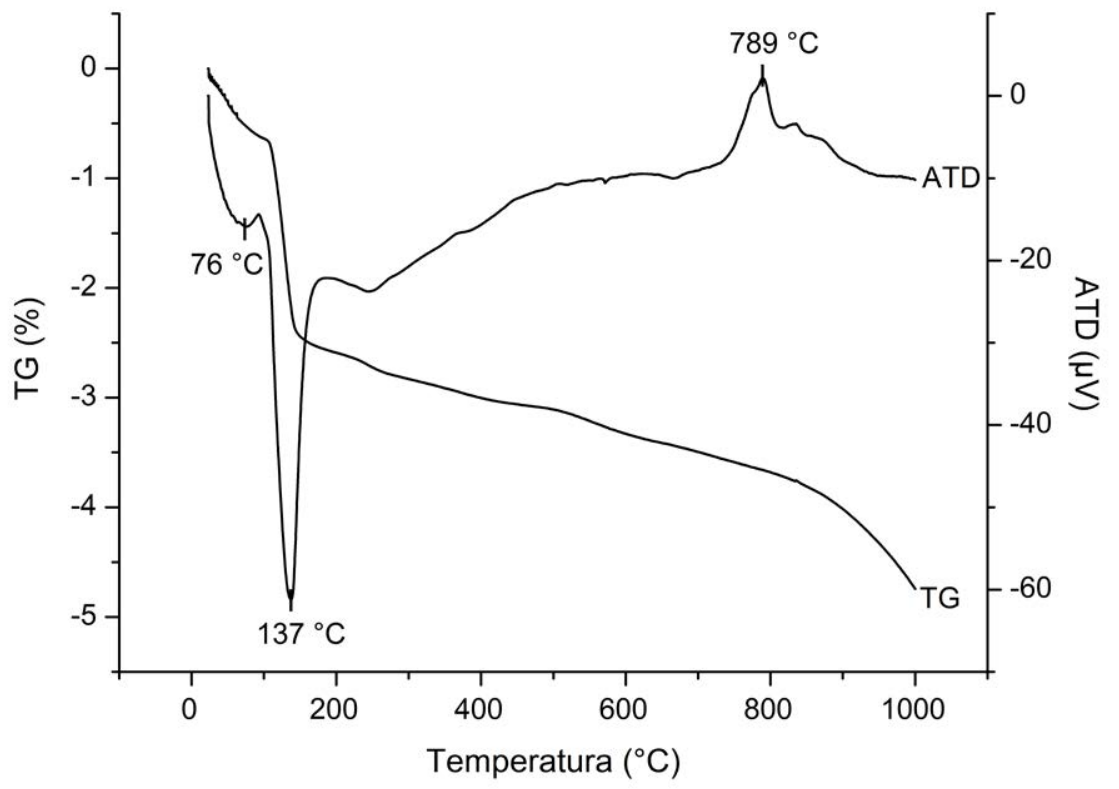

Figura 5. Comportamiento térmico de la escoria.

también puede intervenir en forma de un óxido básico cuando la proporción de sílice es elevada, obteniéndose silicato de aluminio. Dicho producto no fue detectado por DRX en las experiencias realizadas, lo que ratifica de manera inequívoca que la muestra se clasifique químicamente de tipo ácido $(\mathrm{IB}<1)$. Un aumento de la basicidad, con la restricción de que no fuera el resultado del incremento de la concentración de $\mathrm{CaO}$, disminuye la viscosidad de la escoria (Bazán et al., 2006). El segundo de los parámetros fue medido por picnometría y su bajo valor, consistente con la moderada presencia de hierro indicada en Tabla 1, favorece la separación por gravedad de la mata. 


\section{Contaminación atmosférica provocada por la oxidación de enargita, pirita y sulfuro cuproso}

Un aspecto no contemplado por autores locales que trataron la temática de la recuperación del cobre a partir de minerales extraídos de Famatina, fue la contaminación causada por los desprendimientos de dióxido de azufre y óxido arsenioso. No obstante, la situación había sido advertida para el primero de los gases desde hacía más de sesenta años. Así, el metalurgista francés F. Le Play cuantificó en 1840 que las fundiciones ubicadas a lo largo del valle de Swansea (Gales) descargaron en la atmósfera 92.000 ton de $\mathrm{SO}_{2}$. Si bien no fue especificado a que período se adjudicaba dicha emisión, es una magnitud que confirma la importancia del planteo. En cuanto al efecto ambiental negativo del arsénico contenido en los humos de dichos establecimientos, los registros son más recientes (1902) y fueron inicialmente confirmados en Montana (EE. UU.) (Pérez Cebada, 1999). Sintetizando, la problemática en cuestión se conocía al momento de constituirse la empresa que iba a efectuar la explotación de La Mejicana. Actualmente, existe amplio consenso científico académico para evaluar la contaminación antrópica provocada por actividades mineras realizadas hace un siglo o más y que todavía perdura (Cooke et al., 2009; Fernández Caliani, 2008; Fernández Caliani, González, Aparicio, Barba y Galán, 2005; Folchi, 2005; Uglietti, Gabrielli, Cooke, Vallelonga y Thompson, 2015).

Los pesos de $\mathrm{SO}_{2}$ y $\mathrm{As}_{4} \mathrm{O}_{6}$ formados según la ecuaciones ((2)-(3), (6), (9)-(10)) se estimaron mediante un cálculo estequiométrico sencillo. Al superar la temperatura de oxidación $300^{\circ} \mathrm{C}$, la molécula de óxido arsenioso generada fue la del dímero (395,6 g $\mathrm{mol}^{-1}$ ) (Aracena Caipa, 2013; Mihajlovic et al., 2007). Se trabajó con datos de 1911 ya que de allí en adelante se comercializó sólo cobre enriquecido por fusión de la mata. Durante ese año fueron exportados $\sim 644,9$ ton de blíster en lingotes de $40 \mathrm{~kg}$, con una pureza de $98 \%$ p/p de $\mathrm{Cu}$ (Anuario de la Dirección General de Estadísticas-1911, 1912; Leguizamon Pondal, 1914). Si se supone que $\mathrm{Cu}_{3} \mathrm{AsS}_{4}$ es el único mineral portador del metal, se habrían requerido $\sim 1.320$ ton de enargita para lograr la producción antedicha. La reserva estimada de Upulungos duplicaba la de San Pedro, así que de acuerdo con esta relación y sus respectivas leyes se tiene una composición promedio de $4,32 \% \mathrm{p} / \mathrm{p}$ de $\mathrm{Cu}$ (Anónimo, 1910; Hermitte, 1917). Conforme a dicho porcentaje, la cantidad de mena empleada ascendería a $\sim 14.650$ ton.

Para el cómputo se asumió que: 1) Durante el lapso señalado no hubo cambio de tecnología; 2) la pérdida de $\mathrm{Cu}$ en la escoria ha sido despreciable (Tabla 1) al no indicarse de modo explícito la proporción de pirita, su valor fue establecido tomando en cuenta la suma de todo el Fe del recurso: 15,87\% (Leguizamon Pondal, 1914). Conviniendo arbitrariamente que el $25 \% \mathrm{p} / \mathrm{p}$ es de otros componentes - carbonato de hierro, hierro titanífero (Bodenbender, 1916), olivina, serpentina (Lannefors, 1926), etc. - se tiene en la roca beneficiada $11,90 \% \mathrm{p} / \mathrm{p}$ de hierro pirítico $(25,54 \% \mathrm{p} / \mathrm{p}$ de bisulfuro) y equivaldría a $\sim 3.750$ ton de $\mathrm{FeS}_{2}$.

El balance de masa llevado a cabo permitió determinar los desprendimientos de $~ 335$ ton de $\mathrm{As}_{4} \mathrm{O}_{6} \mathrm{y} \sim 540$ ton de $\mathrm{SO}_{2}$ a partir de enargita (ecuación (6)). La otra emisión que se debe contemplar es el $\mathrm{SO}_{2}$ procedente de la oxidación de pirrotina, durante la obtención ya sea de la mata o del blíster (ecuaciones (2) o (3)), y del sulfuro cuproso (ecuaciones (9)-(10)): 2.050 ton y 330 ton, respectivamente. Con relación al FeS, los moles volatilizados de dióxido de azufre son independientes de la valencia del hierro. Asimismo, en la anterior ponderación se acordó que la conversión de $\mathrm{FeS}_{2}$ a FeS fue completa (ecuación (1)) y además no se contabilizó la participación del azufre condensado en las áreas frías del horno $\left(\mathrm{S}_{2}(\mathrm{~g})+2 \mathrm{O}_{2}(\mathrm{~g}) \rightarrow 2 \mathrm{SO}_{2}(\mathrm{~g})\right)$.

Una reflexión final le concierne al $\mathrm{CO}_{2}$ liberado cuando se quemaba materia orgánica $\left(\mathrm{C}(\mathrm{s})+\mathrm{O}_{2}(\mathrm{~g}) \rightarrow \mathrm{CO}_{2} \otimes(\mathrm{g})\right)$. Si bien su consecuencia ambiental no es tan grave como 
la provocada por arsénico y azufre ni su origen se relaciona con el aprovechamiento de un recurso natural específico, se destaca su presencia ya que es uno de los principales causantes del efecto invernadero (Fernández Caliani, 2008; Harte, Holdren, Schneider y Shirley, 1995; Pérez Cebada, 1999).

\section{Breve análisis sobre el impacto de la minería en la desforestación regional}

Como fuente alternativa de la energía proporcionada por carbón y coque a fin de calefaccionar los hornos (Hermitte, 1917; Leguizamon Pondal, 1914, 1919; Wässman, 1926) se usaba leña de zonas aledañas a la planta (Rojas, 2013). Un segundo gasto, aunque considerablemente menor al de la fundición, fue el requerido para accionar los motores a vapor que impulsaban el cable carril (Lannefors, 1926; Rojas, 2013). Los árboles más abundantes de los bosques nativos son algarrobo y retama. La capacidad calorífica para cada tipo de madera depende de su humedad al momento de la combustión. El valor promedio para el algarrobo es de $\sim-19.650 \mathrm{~kJ} \mathrm{~kg}^{-1}$ (Serna Mosquera, Borja de la Rosa, Fuentes Salinas y Corona Ambriz, 2011).

El régimen autógeno (Kuzmín y Samojotski, 1986; Peters, 1907) presume un ahorro enorme de combustible, su ocurrencia se debe al carácter fuertemente exotérmico con que se oxida la pirrotina. Las $\Delta \mathrm{G}_{700^{\circ} \mathrm{C}}$ ya anotadas en $\mathrm{kJ} \mathrm{mol}^{-1}$ (sección Reacciones formuladas para describir la obtención del cobre) son ahora expresadas en otra unidad de masa: $-4.440 \mathrm{~kJ} \mathrm{~kg}^{-1}$ para la ecuación (2) y $\sim-5.410 \mathrm{~kJ} \mathrm{~kg}^{-1}$ para la ecuación (3) (Rosenqvist, 1987). Al compararse con el algarrobo se observa que el aporte energético del sulfuro de hierro es significativo. A pesar de la innegable ventaja de este proceder, no se logró el beneficio esperado (Anónimo, 1910). Luego de los correspondientes ensayos de validación -primeros meses de 1911- se decidió tostar las menas de Upulungos y San Pedro antes de ser cargadas al horno de cuba, con la metodología enunciada en el antedicho apartado (Leguizamon Pondal, 1914, 1919). Las emisiones gaseosas se notaban por las molestias que ocasionaban al respirar (Leguizamon Pondal, 1919; Sola Álvarez, 2012).

Resumiendo, se considera que el consumo para realizar la actividad relatada no fuera tan excesivo como se cree, aun cuando es indudable su contribución al desmonte que tuvo lugar en el oeste de la provincia (Rojas, 2013). La situación se agrava si convenimos que a partir de 1869 funcionaba con intermitencias, cerca de Famatina, un horno industrial para fundir minerales de cobre provenientes de La Mejicana (Hünicken y Hünicken, 2008). Todos los hechos aquí puntualizados acreditan que las sucesivas técnicas implementadas no resultaran sustentables en el tiempo.

Actualmente, para establecer la factibilidad de cualquier proyecto de megaminería, no basta con valorar el comportamiento del metal, la contaminación atmosférica y el consumo de biomasa. Otros puntos que también deben ser evaluados -el listado no es taxativo- son: agua superficial/subterránea, suelo, fauna, flora e inclusive hasta el manejo territorial (Lorca y Hufty, 2017; Sola Álvarez, 2012; Wagner, 2016).

\section{Causales que habrían impulsado la decisión de abandonar definitivamente la explotación del complejo minero metalúrgico}

Durante 1912 The Famatina Development Corporation se transformó en The Famatina Company y al año siguiente, junio de 1913, la compañía sustituta abandonó definitivamente el país (Hermitte, 1917). Entre los pasivos de la empresa, se destacan las deudas al Banco de la Nación Argentina por créditos impagos y a Ferrocarriles del Estado por la utilización del cable carril (Bazán, 2017; Berensztein et al., 2016). Un experto 
en la especialidad sugirió que el cese de actividades se debió a la toma de decisiones metalúrgicas equivocadas y a errores en la construcción de los hornos (Hermitte, 1917). Sin embargo, dichas causales no parecen razonables ya que a comienzos del siglo pasado Inglaterra era un líder mundial en el desarrollo de tecnologías para producir cobre por vía pirometalúrgica (Pérez Cebada, 1999). Una nota de divulgación publicada con antelación a los hechos que nos ocupan, y sustentada en fuentes fidedignas, ya hablaba sobre la existencia de inconvenientes operativos que serían solucionados a la brevedad (Anónimo, 1910).

Hoy en día, se suele asociar la mencionada renuncia a continuar el proyecto con el estallido de la Primera Guerra Mundial (Bazán, 2017; Berensztein et al., 2016; Rojas, 2013; Sola Álvarez, 2012). Llama la atención que el artificio del cambio de firma se haya desencadenado dos años antes de iniciado el conflicto bélico, fines de julio de 1914. Sintetizando, los dos argumentos brindados (actual y pasado) no dilucidarían de modo totalmente satisfactorio la controversia planteada. Una situación conocida en su momento, pero no considerada crítica, habría contribuido a tornar inviable la extracción de minerales de La Mejicana. Temperaturas extremas en invierno (promedio $-6 /-10^{\circ} \mathrm{C}$ ), precariedad de trabajo en las galerías y respiración anormal por la altura, fueron algunas de las condiciones ambientales difíciles de superar. De lo expuesto se deduce que sólo los lugareños podían llevar a cabo la tarea sin padecer demasiadas contrariedades (Bialet Massé, 1904; Leguizamon Pondal, 1914).

La consulta del Anuario de la Dirección General de Estadísticas de la Nación-1911 (1912) no aclaró las dudas, por el contrario se potenciaron. Los datos revisados detallan el comportamiento previo al inicio de la concertada maniobra empresarial. En el capítulo "Exportación-productos de minería" se consigna "cobre en barras" (punto 134): aforo medio $\mathrm{m} \$ \mathrm{n} 0,40$ por $\mathrm{kg}$, cantidad $644.888 \mathrm{~kg}$ y valor de plaza $\mathrm{m} \$ \mathrm{n}$ $257.955(\mathrm{~m} \$ \mathrm{n}=$ símbolo monetario del peso moneda nacional vigente en esa fecha). Lo más sorprendente del registro es que no fue explicitada la presencia de oro y plata en el blíster, cuya composición y cotización de mercado son exhibidas en la Tabla 3 (Kussmaul, 2007; Leguizamon Pondal, 1914, 1919; Wässman, 1926). En relación con lo anterior, se advierte la subvaloración del cobre y se considera indudable que la rentabilidad del emprendimiento dependía enormemente de ambos metales preciosos. Las leyes de estos componentes disminuyeron a medida que avanzaba la explotación de los filones y así el proceso se tornaba menos rentable (Bodenbender, 1916; Hermitte, 1917; Lannefors, 1926; Leguizamon Pondal, 1914; Wässman, 1926). En nuestra opinión, las dificultades intrínsecas del laboreo y el empobrecimiento de la mena evidenciarían de modo más certero lo acontecido.

Para concluir la discusión acerca del tema económico, se accedió al Anuario de la Dirección General de Estadísticas-1908 (1909). De las tres fuentes citadas en la Tabla 2 , surge que durante ese año fue comercializada exclusivamente mata $\left(\mathrm{Cu}_{2} \mathrm{~S}+\mathrm{FeS}+\right.$ trazas de metales preciosos). En el punto 129, "cobre en barras" figura también m \$n 0,40 por kg. La publicación del mismo aforo en sendos documentos oficiales implica que la mejora en la riqueza del producto final no condujo a ninguna compensación monetaria.

Tras cinco años de interrupción, aunque sólo por un período breve (1918-1923), se volvió a trabajar en la mina y en Santa Florentina. La Corporación Minera Famatina, de origen nacional, tuvo a su cargo la reapertura del complejo minero metalúrgico (Angelelli, 1984; Bazán, 2017; Lannefors, 1926; Wässman, 1926). Hacia 1939 se comenzaron nuevas exploraciones en La Mejicana con resultados inconducentes. Más tarde hubo otro intento que también fracasó (Angelelli, 1984; Bazán, 2017). Luego de que la planta dejara de operar (1923) fue conservada hasta mediados de la década siguiente, pero con el correr del tiempo el sector de los hornos fue desmantelado. Desde hace más de medio siglo las instalaciones están totalmente inutilizables y la chimenea, 


\begin{tabular}{|l|c|c|c|c|}
\hline Elemento & $\%(\mathrm{p} / \mathrm{p})$ & Cantidad $(\mathrm{kg})$ & $\mathrm{m} \$ \mathbf{n} / \mathrm{kg}$ & $\mathrm{m}$ \$़ \\
\hline $\mathrm{Cu}$ & 98,00 & 631.990 & 0,70 & 442.390 \\
$\mathrm{Ag}$ & 0,80 & 5.159 & 32,96 & 170.020 \\
$\mathrm{Au}$ & 0,17 & 1.096 & $1.225,65$ & 1.343 .314 \\
$\mathrm{~S}$ & 1,03 & 6.643 & - & - \\
\hline Total & 100,00 & $\mathbf{6 4 4 . 8 8 8}$ & - & $\mathbf{1 . 9 5 5 . 7 2 4}$ \\
\hline
\end{tabular}

Tabla 3. Composición química del blíster producido en 1911 y precio de venta de cada metal extraído (Leguizamon Pondal, 1914; Lannefors, 1926).

aún sigue en pie, es una imagen que por sí misma ilustra la magnificencia de aquel establecimiento industrial (Figura 2).

\section{Consideraciones finales}

El constituyente de la mena reportado como principal especie portadora de cobre fue enargita y su oxidación, ejecutada insuflando suficiente aire, habría provocado la descarga en la atmósfera de apreciables cantidades de óxido arsenioso y dióxido de azufre. Un porcentaje muy pequeño del referido metal en comparación con las leyes de ambas vetas explotadas -Upulungos y San Pedro- quedó retenido en la escoria, fase menos densa de la masa fundida. Por esta razón, se califica de eficaz al sistema operativo utilizado. La problemática del laboreo y una disminución de la riqueza de metales preciosos en la mena habrían sido dos causales decisivos al momento de interrumpir el proyecto.

Se comprobó la preponderancia del aporte energético de $\mathrm{FeS}_{2}$, una de las impurezas mayoritarias del yacimiento, que posibilitaba disminuir el dispendio del recurso forestal regional. Sin embargo, el producto volátil generado superaba apreciablemente en peso a la suma de los dos anteriores. Las pruebas metalúrgicas efectuadas por la empresa propietaria de la mina desaconsejaron la fundición pirítica en el circuito productivo empleado.

En la escoria se identificó yeso, $\alpha$-cuarzo, monticellita y augita. La primera sustancia se infiere fue formada por reacción química y la segunda fue uno de los fundentes agregados. Con relación a los silicatos, su origen respondería al propósito de sintetizar productos con bajo punto de fusión. En tanto, no fue detectada la presencia de wüstita, hematita, fayalita y magnetita. Por efecto térmico sobrevino la conversión paulatina del estado ferroso a férrico, obteniéndose $\mathrm{Fe}_{2} \mathrm{O}_{3}$ cristalino. Se determinó que la muestra examinada presumiblemente contenía carbono, atribuyéndose su procedencia a la posible re carbonatación del material fundido durante el enfriamiento. Finalmente, el estudio arqueométrico realizado representa una vía capaz de dilucidar cuestiones básicas de la tecnología para extraer cobre que fuera aplicada hace más de 100 años en el establecimiento Santa Florentina.

\section{Agradecimientos}

Los autores agradecen a N. Hugo Fava, Lucas R. Huck (CETMIC) y Elba N. Saenz por la colaboración recibida en el desarrollo del trabajo. Asimismo, extienden su reconocimiento a los evaluadores y editores que mediante las oportunas observaciones hechas permitieron mejorar el manuscrito. Este estudio fue financiado por la Comisión de Investigaciones Científicas de la Provincia de Buenos Aires y el Consejo Nacional de Investigaciones Científicas y Técnicas. 


\section{Deferencias citadas}

"Angelelli, V. (1984). Yacimientos metaliferos de la República Argentina. La Plata: Comisión de Investigaciones Científicas de la Provincia de Buenos Aires.

"Anónimo. (1910). Famatina Development Corporation. The Mining Magazine, III, 375376. https://archive.org/details/miningmagazineo3londuoft/page/ (Acceso: 23 de mayo, 2019).

»Anónimo. (1914). Mercado de cobre en 1912. Boletín de la Sociedad Nacional de Minería, Año XXXI, Vol. XXVI, Serie III, 85. http://www.sonami.cl/digital/boletin/o203_1914_01/ (Acceso: 23 de mayo, 2019).

» Anuario de la Dirección General de Estadísticas, Correspondiente al año 1908. (1909). En 129 - Cobre en barras, Tomo 1 (p. 567). Buenos Aires: Compañía Sud-Americana de Billetes de Banco.

" Anuario de la Dirección General de Estadísticas, Correspondiente al año 1911. (1912). En 134 - Cobre en barras, Tomo 1 (p. 461). Buenos Aires: Talleres de Publicaciones de la Oficina Meteorológica Argentina.

" Aracena Caipa, A. G. (2013). Mecanismo y cinética de oxidación de enargita $\left(\mathrm{Cu}_{3} \mathrm{AsS}_{4}\right)$ y estibina $\left(\mathrm{Sb}_{2} \mathrm{~S}_{3}\right.$ ) a altas temperaturas. (Tesis de Doctorado inédita), Facultad de Ingeniería, Universidad de Concepción, Chile.

" Barone, V. L., Gazzoli, D., Lick, I. D., Schalamuk, I. B. y Botto, I. L. (2011). Thermal and spectroscopic feature of the $\mathrm{Cu}_{3} \mathrm{AsS}_{4}$ enargite oxidation up to $800{ }^{\circ} \mathrm{C}$. Implications in the arsenic evolution. Asian Journal of Research in Chemistry, 4(12), 1844-1850.

» Bazán, R. E. (2017). Cuatro siglos de minería en La Rioja, Argentina. (Tesis de Maestría inédita), Facultad de Ciencias Económicas, Universidad de Buenos Aires, Argentina.

» Bazán, V., Goñi, Ch., Castellá, L., Brandaleze, E., Verdeja, L. F. y Parra, R. (2006). Estimación de la viscosidad de escorias fayalíticas utilizando el modelo de cálculo kv y el método experimental del plano inclinado. Revista de Metalurgia, 42(2), 84-90.

" Becerra, M. F., Nieva, N. y Angiorama, C. I. (2014). Hornos, minerales y escorias: una aproximación a la metalurgia extractiva en la puna de Jujuy en época prehispánica y colonial. Arqueología, 20(1), 13-29.

"Berensztein, S., Secco, L., Depetris, R., Duer, W., Nigro, M. y Rumi, C. (2016). Banco Nación Argentina: los primeros 125 años (p. 58). Ciudad Autónoma de Buenos Aires: Banco de la Nación Argentina.

»Bialet Massé, J. (1904). La Rioja. La minería - La alta agricultura. En Informe sobre el estado de las clases obreras en el interior de la República, Tomo Primero, Capítulo VII (pp. 225-321). Buenos Aires: Imprenta y Casa Editora de Adolfo Grau.

»Bodenbender, G. (1916). El nevado de Famatina. Boletín de la Academia Nacional de Ciencias, Tomo XXI, 100-182.

»Brodtkorb, M. K. de y Schalamuk, I. B. (1999). Yacimientos de cobre y oro de la Sierra de Famatina, La Rioja. En E. O. Zappettini (Ed.), Recursos Minerales de la República Argentina, Anales 35 (pp. 1659-1663). Buenos Aires: Instituto de Geología y Recursos Minerales, SEGEMAR.

" Cooke, C. A., Wolfe, A. P. y Hobbs, W. O. (2009). Lake-sediment geochemistry reveals 1400 years of evolving extractive metallurgy at Cerro de Pasco, Peruvian Andes. Geology, 37(11), 1019-1022. 
"Díaz Marinovic, J. I. (2012). Cinética de conversión de mata de cobre y distribución de impurezas en lecho empacado. (Tesis de Maestría inédita), Facultad de Ciencias Físicas y Matemáticas, Universidad de Chile, Chile.

»Evans, C. y Saunders, O. (2017). El mundo del cobre en el siglo XIX: una introducción. Revista de Historia Social y de las Mentalidades, 21(1), 9-26.

» Fernández Caliani, J. C. (2008). Una aproximación al conocimiento del impacto ambiental de la minería en la faja pirítica Ibérica. MACLA: Revista de la Sociedad Española de Mineralogía, 10, 24-28.

» Fernández Caliani, J. C., González, I., Aparicio, P., Barba, C. y Galán, E. (2005). Niveles de concentración de arsénico y metales pesados en los suelos del entorno de las minas abandonadas de la faja pirítica Ibérica. MACLA: Revista de la Sociedad Española de Mineralogía, 3, 73-74.

" Folchi, M. (2005). Los efectos ambientales del beneficio de minerales metálicos: un marco de análisis para la historia ambiental. Varia Historia, 33, 32-57.

» Gaviría, J. P., Quattrini, D., Fouga, G., Bohé, A., y Pasquevich, D. (2005). Estudio cinético de la descomposición del carbonato de calcio por cromatografía gaseosa. Revista Matéria, 10(1), 170-177.

» Gill, C. B. (1989). Metalurgia extractiva no ferrosa. México: Editorial Limusa.

» Hammond, C. R. (1981). Physical constants of inorganic compounds. En R.C. Weast (Ed.), CRC Handbook of Chemistry and Physics (pp. B81-B131). Boca Ratón, Florida: CRC Press.

" Harte, J, Holdren, C., Schneider, R. y Shirley, C. (1995). Guía de las sustancias contaminantes. México: Editorial Grijalbo.

» Hermitte, E. (1917). Minerales de cobre. La geología y minería argentina en 1914. En Tercer censo nacional de la República Argentina, Censo de las Industrias, Tomo VII (pp. 469-476). Buenos Aires: Talleres Gráficos de L. J. Rosso y Cía.

» Hünicken, M. y Hünicken, H. (2008). Contribución de Emilio Hünicken en el inicio de la Minería y Geología en la Argentina. Serie Correlación Geológica, 24, 85-90.

" Jovanovic, D. (1989). Kinetics of thermal decomposition of pyrite in an inert atmosphere. Journal of Thermal Analysis, 35(5), 1483-1492.

" Kussmaul, S. (2007). Publicaciones de principios del siglo XX sobre las minas de oro en Costa Rica. Revista Geológica de América Central, 36 Especial, 115-123.

» Kuzmín, B. A. y Samojotski, A. I. (1986). Capítulo 16. En Metalurgia, metalografía y materiales de construcción, Cobre y sus aleaciones (pp. 191-198). Moscú: MIR.

"Lannefors, N. A. (1926). I. Sobre las minas de cobre de Famatina. Publicación № 21. Buenos Aires: Dirección General de Minas, Geología e Hidrología.

"Leguizamon Pondal, M. (1914). Riquezas minerales del Famatina. Revistas de la Universidad de Buenos Aires, Año XI, Tomo XXV, 257-275.

» Leguizamon Pondal, M. (1919). Metalurgia de los minerales del Famatina. Boletín Minero de la Sociedad Nacional de Minería, Santiago, Chile, Serie III, 240, 159-169.

» Lorca M. y Hufty, M. (2017). El patrimonio como forma de resistencia a la gran minería. El caso del Huasco Alto, Chile. Intersecciones en Antropología, 18(1), 31-42.

» Mackenzie, R. C. (Ed.). (1970). Differential thermal analysis. Londres: Academic Press.

» Menéndez, C. J., Tavani, E. L. y Nolasco, E. J. (2003). Determination of kinetic aspects from the non-isothermal chlorination of tungsten minerals. Latin American Applied Research, 33(1), 19-26. 
» Mihajlovic, I., Strbac, N., Zivkovic, Z., Kovacevic, R. y Stehernik, M. (2007). A potential method for arsenic removal from copper concentrates. Minerals Engineering, 20(1), 26-33.

»Nazer, A., Payá, J., Borrachero, M. V. y Monzó, M. (2016). Caracterización de escorias de cobre de fundiciones chilenas del Siglo XIX. Revista de Metalurgia, 52(4), 1-9.

»Ortega, L. A., Zuluaga, M. C., Alonso, A. y Olaetxea, C. (2005). El estudio arqueométrico de las producciones cerámicas. Munibe Antropologia-Arkeologia, 57, 365-388.

» Pérez Cebada, J. D. (1999). Minería del cobre y contaminación atmosférica. Estrategias empresariales en las cuencas de Swansea, Huelva y Montana. Revista de Historia Industrial, 16, 45-67.

» Peters, E. D. (1907). The principles of copper smelting. Nueva York: Hill Publishing Company.

»Quiroga, M., Chiavazza, H., Lascalea, G. y Gurrito, M. (2017). Estudios arqueométricos de clavos procedentes de contextos funerarios de las ruinas de San Francisco (Mendoza, Argentina). Arqueología, 23(1), 45-60.

»Rendtorff, N. M., Morosi, M. E. y Tavani, E. L. (2018). Análisis del proceso empleado para la extracción de cobre a partir de minerales del yacimiento 'La Mejicana', Chilecito, Argentina. En C. Baied, C. Somonte, M. Maloberti y M. L. Cohen (Eds.), Libro de Resúmenes Extendidos del VII Congreso Nacional de Arqueometría (pp. 171176). Tucumán: Facultad de Ciencias Naturales e Instituto Miguel Lillo, Universidad Nacional de Tucumán.

» Rojas, F. (2013). Rol de la minería y el ferrocarril en el desmonte del oeste riojano y catamarqueño (Argentina) en el período 1851-1942. Población \& Sociedad, 20(2), 99-123.

» Rosenqvist, T. (1987). Fundamentos de metalurgia extractiva. México: Editorial Limusa.

» Rovira, S. y Burillo, F. (2005). Experimentos de fundición de minerales de hierro en la ciudad Celtibérica de Segeda (Mara, Zaragoza). En J. Molera Marimon, J. Farjas Silva, P. Roura Grabulosa y T. Pradell Cara (Eds.), Actas del VI Congreso Ibérico de Arqueometría (pp. 137-143). Girona, España: Universitat de Girona.

»Serna Mosquera, Y. B., Borja de la Rosa, A., Fuentes Salinas, M. y Corona Ambriz, A. (2011). Propiedades tecnológicas de la madera de algarrobo (hymenaea oblongifolia huber.), de Bagadó-Chocó, Colombia. Revista Chapingo, Serie Ciencias Forestales y del Ambiente, 17(3), 411-422.

»Setti, M., Lanfranchi, A., Cultrone, G. y Marinoni, L. (2012). Estudio arqueométrico y evaluación del deterioro de los materiales cerámicos de la fachada de la iglesia de Santa Maria del Carmine (Pavia, Italia). Materiales de Construcción, 62(305), 79-98.

»Sola Álvarez, M. (2012). Conflictos socioambientales en torno a la megaminería metalífera a cielo abierto. (Tesis de Maestría inédita), Facultad de Arquitectura, Diseño y Urbanismo, Universidad de Buenos Aires, Argentina.

»Szczygiel Jordens, Z. y Torres Reyes, A. (1984). Metalurgia no ferrosa. México: Limusa.

» Thibodeau, A. M., Killick, D. J., Ruiz, J., Chesley, J. T., Deagan, K., Cruxent, J. M. y Lyman, W. (2007). The strange case of the earliest silver extraction by european colonists in the new world. Proceedings of the National Academy of Sciences, 104(9), 3663-3666.

»Uglietti, C., Gabrielli, P., Cooke, C. A., Vallelonga, P. y Thompson, L. G. (2015). Widespread pollution of the South American atmosphere predates the industrial revolution by $240 \mathrm{y}$. Proceedings of the National Academy of Sciences, 112(8), 2349-2354.

» Varela, O., Parrado, M. F. y Buedo, S. E. (2015). Diversidad de plantas vasculares del valle Antinaco-Los Colorados, La Rioja, Argentina. Boletín de la Sociedad Argentina de Botánica, 50(3), 385-411. 
"Vera Giglio, P. A. (2007). Cinética de oxidación de mata industrial de cobre con formación de escoria olivina. (Tesis de Grado inédita), Facultad de Ciencias Físicas y Matemáticas, Universidad de Chile, Chile.

»Wagner, L. (2016). Conflictos socioambientales por megaminería en Argentina: apuntes para una reflexión en perspectiva histórica. AREAS, Revista Internacional de Ciencias Sociales, 35, 87-99.

»Wässman, S. (1926). II. Establecimiento metalúrgico de Santa Florentina. Publicación Nº 21. Buenos Aires: Dirección General de Minas, Geología e Hidrología. 